\title{
Kidneys, Hearts, Hormones and Immunomodulators: Integrated Understandings
}

\author{
Adeera Levin \\ University of British Columbia, St. Paul's Hospital, Vancouver, Canada
}

\section{Key Words}

Chronic kidney disease $\cdot$ Cardiovascular disease .

Renin-angiotensin system

\begin{abstract}
The biological processes governing vascular endothelial cell health are complex and highly redundant. The mechanisms involved in cell injury and repair remain an area of intense study. This paper attempts to review current knowledge regarding hormones such as vitamin D, erythropoietin and aldosterone within the context of what we understand of the inflammatory cascade. This is not a comprehensive review of vascular biology, and many important details and background information have been omitted due to space and complexity considerations. The integration of exocrine and endocrine functions within the kidney, the intimate relationship of those functions with vascular health, and the clinical observations of high prevalence of cardiovascular disease in chronic kidney disease warrant a better understanding of this area by clinicians and basic researchers.
\end{abstract}

Copyright (C) 2006 S. Karger AG, Basel

\section{Introduction}

Chronic kidney disease (CKD) is increasingly recognized as being prevalent in all countries. CKD is associated with adverse cardiovascular disease (CVD) outcomes, and CKD patients are more likely to die, predominantly of $\mathrm{CV}$ events, than to reach end-stage kidney disease [1-3]. Factors that contribute to both progressive kidney disease and the associated cardiovascular morbidity have been increasingly identified and are often similar. The complex biological processes associated with both conditions share a common target: the vascular bed. Thus, the coincident existence of both conditions is more readily explainable.

This paper attempts to describe the current understanding of the pathophysiology of CVD in CKD, with specific focus on new insights into hormonal, immunomodulatory and non-traditional risk factors, and most specifically, the interaction between factors. The integration of understanding basic processes and their translation into clinical strategies is important if we are to impact the outcomes of individuals living with kidney disease.

The biological processes that are involved in progressive CKD and CVD center around cell damage which is incited by and incites inflammation, which subsequently leads to fibrosis, apoptosis and cell repair. Overall, recurrent damage leads to dysregulated balance between the

\section{KARGER}

Fax +4161306 1234 E-Mail karger@karger.ch www.karger.com
Prof. Adeera Levin, MD, FRCPC

University of British Columbia, St. Paul's Hospital

1081 Burrard St., Rm 6010-A

Vancouver BC, V6Z1Y8 (Canada)

Fax +1 604806 8120, E-Mail alevin@providencehealth.bc.ca 
complex systems of inflammation and repair, such that progressive kidney function decline occurs. It is intriguing that many of the hormonal and cytokine derangements described in kidney disease can be viewed as both causal and the effect of loss of kidney function. The vascular disease that characterizes progressive CKD is not limited to the glomerulus, but also involved other vascular beds within the individuals. Thus, the processes mitigating progressive kidney damage are most likely those contributing to excessive cardiovascular disease.

In addition to the usual factors contributing to pathological processes (hypertension, diabetes, dyslipidemia), it is becoming evident that specific factors such as hormones, chemokines and cytokines are important. The key players in these processes include hormones, such as angiotensin II, aldosterone, cortisal, vitamin D and erythropoietin, inflammatory cytokines, such as interleukin (IL)-6, transforming growth factor- $\beta$, tumor necrosis factor- $\alpha$ and $\mathrm{C}$-reactive protein, and adhesion molecules, such as vascular cell adhesion molecule and intracellular adhesion molecule. In addition, proteins involved in reparative processes such as bone morphogenic protein-7, growth factor and insulin-derived growth factor have also been described in the context of progressive CVD and CKD

It is beyond the scope of this paper to describe in detail all the factors associated with progressive CKD and CVD; specifically, we will not discuss hypertension, proteinuria and dyslipidemia per se. Furthermore, there is extensive information on each of the hormones, cytokines and chemokines, which cannot be covered. However, it is our intention to describe key interactions that may be of specific clinical relevance, with a focus on vitamin $\mathrm{D}$, aldosterone and erythropoietin as key hormones and immunoregulators of essential cell functions.

\section{Consequences of Kidney Damage and Reduced Glomerular Filtration Rate}

Low glomerular filtration rate (GFR) confers a poor outcome in numerous populations studied [3]. Most of the observational data gleaned from diverse or selected populations have relied on limited data from administrative data sets. Thus, while it is well known that the prevalence of associated abnormalities, such as hemoglobin, calcium, phosphate, parathyroid hormone (PTH), blood pressure and inflammatory markers, are altered in CKD [4-9], the complexity of the impact of those alterations is underappreciated and undertreated.

Kidneys, Hearts, Hormones and Immunomodulators
In patients with $\mathrm{CKD}$, both atherosclerotic and arteriosclerotic processes occur, triggered by different events, but ultimately culminating in impaired endothelial cell function. While ischemic disease is certainly prevalent, stiffening of the arterial vessels leading to an increase in left ventricular stress and pressure, subsequent hypertrophy, and ultimately, cardiomyopathy contributes to the high prevalence of heart failure and sudden death seen in CKD populations. The atherosclerotic process has recently been described as an inflammatory process in which endothelial damage incites a cascade of events ultimately leading to plaque formation [10-13]. Importantly, CKD patients irrespective of stage have evidence of activated inflammatory processes both prior to and on dialysis. This inflammatory state of uremia is likely the cornerstone of progressive vascular disease.

The arteriosclerotic process is driven by a combination of factors including aging, collagen loss, apoptosis, and subsequently, medial calcification, all of which lead to increased vessel stiffness [14, 15]. These processes appear to be exacerbated, or perhaps even initiated, in the context of inflammation, but are driven by a series of events which are biochemically and hormonally mediated.

Reduction in GFR is accompanied quite early on by reduced levels of a number of hormones and dysregulation of processes which are important in cell repair. Specifically, vitamin D production/hydroxylation to active forms is reduced, loss of erythropoietin synthesis occurs, and activation of the renin angiotensin aldosterone system is evident.

\section{Vitamin D}

Vitamin D has recently been determined to have a number of non-bone-related actions. Specifically, it has been shown to be an inhibitor of various aspects of inflammation, an antiproliferative hormone for myocardial cell hypertrophy, and a negative endocrine regulator of the renin-angiotensin system (RAS) [16, 17].

Vitamin D receptors (VDRs) are found in all organs, including the heart, vascular walls, kidney and immune cells. The VDR is a ligand-activated transcriptional factor which regulates a number of genes concerned with calcium phosphate and other physiologic processes as well.

The inflammatory response which culminates in cytokine release (IL-1, IL-4, IL-6, TNF- $\alpha$ ) by macrophages and $\mathrm{T}$ cells induces smooth muscle cell proliferation and plaque formation. All of these processes can be inhibited 
by vitamin $\mathrm{D}$ and stimulated by high $\mathrm{PTH}$ and high phosphate. Interestingly, vitamin D is immunoregulatory in that it can downregulate inflammatory cells and upregulate anti-inflammatory cells (e.g., IL-10), such that an anti-inflammatory cytokine profile is produced [18].

Vitamin D also modulates the expression of tissue matrix metalloproteinases (MMPs), which are connective tissue enzymes secreted by activated macrophages during inflammation. MMPs are involved in vascular remodeling. Interestingly, MMP-9 levels have been inversely correlated to vitamin D levels [19]. Low vitamin D levels have also been associated with increased cardiac calcification, and higher levels of vitamin D with lower C-reactive protein and higher endothelial progenitor cell levels. Small clinical studies have described the regression of left ventricular hypertrophy in hemodialysis patients with administration of vitamin $\mathrm{D}[20,21]$. This observation is supported by cell data describing the inhibition of cell proliferation in neonatal rat myocytes with activated preparation of vitamin $\mathrm{D}$.

Lastly, vitamin D deficiency has been described in association with hypertension and high renin states. In mice models of VDR knock-out mice, higher levels of renin and angiotensin II have been described in association with left ventricular hypertrophy and hypertension [2226].

Thus, it appears that vitamin D deficiency, a frequent accompaniment of early kidney disease, and loss of GFR may have a number of key roles in the pathogenesis of CVD.

\section{Erythropoietin}

The extrahematological effects of erythropoietins have been well described in recent publications [27, 28]. As an antiapoptotic, anti-inflammatory hormone, it is important in the regulation of the balance of cell injury and repair. Erythropoietin deficiency has been linked to reduced red cell survival, vascular health, neuronal health, renal tubular injury during ischemia and impaired neovascularization. In whole organism and patient studies, erythropoietin is important in maintaining red cell mass, cognitive function, exercise capacity, and possibly, protecting vital organs against ischemic damage.

Of late, the relationship between vitamin D and anemia has been described in a number of associative or small studies. In the presence of vitamin D, erythropoietic hormone requirements are reduced, and hemoglobin levels rise. This effect appears most prominent in patients with elevated PTH, but has been described across the spectrum. Given the previous discussions on anti-inflammatory properties of vascular disease and the known erythropoietin resistance in the presence of inflammation and hyperparathyroidism, this relationship warrants further careful study.

\section{Aldosterone and Activation of the RAS}

While the activation of the RAS system has long been known to adversely impact both kidneys and hearts, the specific action of each component has been more recently described. Given the plethora of information available on angiotensin inhibition and the widespread use of both angiotensin-converting enzyme inhibitors and angiotensin receptor blockers, we will focus on a less often discussed member of the cascade, namely aldosterone.

The role for aldosterone as a deleterious hormone for CVD has been described, specifically as a potent inducer of myocardial fibrosis, and the use of antagonists for patients with symptomatic heart failure is recommended [29-31].

There is experimental evidence that aldosterone contributes to the development of glomerulosclerosis. Quinkler et al. [31] have quantified a number of aspects of aldosterone action in human biopsies and determined that aldosterone/mineralcorticoid receptor activation is related to inflammation and proteinuria.

Interestingly, McCarty [32] has recently hypothesized that through complex mechanisms, high protein diets leading to metabolic acidosis may contribute to hypercortisol and hyperaldosterone states. These in turn may promote visceral obesity and ultimately increase insulin resistance and the potential for type II diabetes. Metabolic acidosis promotes excretion of hydrogen ion via the induction of glutamine, which secondarily increases adrenocorticotropic hormone levels. It is these higher levels of adrenocorticotropic hormone which may lead to a cascade of hormonal events as described above, designed in the first instance to improve ammonia excretion. This fascinating hypothesis adds credence to the importance of low protein diets as being protective for patients with CKD.

\section{Cytokines and Chemokines}

The role of various cytokines and biomarkers which describe the extent of cell injury have been the topic of multiple reviews and original research in the recent past. 
The reader is referred to excellent papers by Stenvinkel [13] and Zoccali et al. [33-35], which describe associations of specific biomarkers of inflammation with patient outcomes.

\section{Clinical Implications}

To date, most clinical studies have focused on the modification of the 'effects' of various hormones and processes. The focus has been on the endpoints of hormonal function (PTH level, hemoglobin level) instead of determining the complex interaction between the processes that regulate these easily measurable factors. Recent data suggest that the administration of vitamin D, irrespective of calcium phosphate and PTH levels, results in better outcomes [36, 37]; further, that patients receiving erythropoiesis-stimulating agents have better outcomes than those who do not, and that those who respond to erythropoiesisstimulating agents with hemoglobin $>12 \mathrm{~g} / \mathrm{dl}$ have better outcomes [38]. Patients who have achieved best blood pressures and blockage of the RAS also have improved outcomes both prior to and on dialysis [39-41].

In the context of current knowledge, we hypothesize that the imbalance of essential hormonal processes which impact on immunodulatory cytokine and chemokine release leads to an unchecked state of inflammation, which propagates ongoing vascular disease. Subsequent abnor- malities within the uremic milieu (including lack of inhibitors of calcification, supersaturation with various compounds and ongoing shear stress/vascular endothelial cell injury) further propagate vascular disease.

The exocrine and endocrine functions of the kidney have been well described for years, but the interaction of loss of hormone synthesis and loss of GFR has not been well studied in the whole organism with respect to the direct and indirect impact on vascular health. A unifying hypothesis that explains the poor outcomes of patients with low GFR would be the loss of internal regulation of processes related to cell injury and repair. This simplification of complex processes is an attempt to bring some rational approach to the care of patients with lower GFR.

It may be of key importance in individual patients to evaluate the extent of hormonal deficiency and inflammatory system activation, of course in the context of easily measured clinical parameters such as GFR level and blood pressure control. Perhaps, a few key medications which restore the internal balance (vitamin $\mathrm{D}$, erythropoietin, aldosterone blockers, RAS blockers and/or anti-inflammatories) should be considered first-line treatment, in conjunction with appropriate diet restriction of key inciters of cell damage (protein/acid load and phosphate). A clearer understanding of the relative importance of each of these factors/strategies at each stage of CKD is important if we are to reduce the burden of CVD and CKD.

\section{References}

1 Weiner DE, Tighiouart H, Stark PC, et al: Kidney disease as a risk factor for recurrent cardiovascular disease and mortality. Am J Kidney Dis 2004;44:198-206.

-2 Weiner DE, Tighiouart H, Amin MG, et al: Chronic kidney disease as a risk factor for cardiovascular disease and all-cause mortality: a pooled analysis of community-based studies. $\mathrm{J}$ Am Soc Nephrol 2004;15:1307-1315.

- 3 Go AS, Chertow GM, Fan D, McCulloch CE, Hsu CY: Chronic kidney disease and the risks of death, cardiovascular events, and hospitalization. N Engl J Med 2004;351:1296-1305.

-4 Clase CM, Garg AX, Kiberd BA: Prevalence of low glomerular filtration rate in nondiabetic Americans: Third National Health and Nutrition Examination Survey (NHANES III). J Am Soc Nephrol 2002;13:1338-1349.

$\checkmark 5$ Coresh J, Astor BC, Greene T, Eknoyan G, Levey AS: Prevalence of chronic kidney disease and decreased kidney function in the adult US population: Third National Health and Nutrition Examination Survey. Am J Kidney Dis 2003;41:1-12.
6 Coresh J, Wei GL, McQuillan G, et al: Prevalence of high blood pressure and elevated serum creatinine level in the United States: findings from the Third National Health and Nutrition Examination Survey (1988-1994). Arch Intern Med 2001;161:1207-1216.

7 Longenecker JC, Coresh J, Powe NR, et al: Traditional cardiovascular disease risk factors in dialysis patients compared with the general population: the CHOICE Study. J Am Soc Nephrol 2002;13:1918-1927.

-8 Hsu CY, McCulloch CE, Curhan GC: Epidemiology of anemia associated with chronic renal insufficiency among adults in the United States: results from the Third National Health and Nutrition Examination Survey. J Am Soc Nephrol 2002;13:504-510.

9 Nickolas TL, Frisch GD, Opotowsky AR, Arons R, Radhakrishnan J: Awareness of kidney disease in the US population: findings from the National Health and Nutrition Examination Survey (NHANES) 1999 to 2000. Am J Kidney Dis 2004;44:185-197.
10 Himmelfarb J, Stenvinkel P, Ikizler TA, Hakim RM: The elephant in uremia: oxidant stress as a unifying concept of cardiovascular disease in uremia. Kidney Int 2002;62:15241538.

11 Zoccali C, Benedetto FA, Mallamaci F, et al: Inflammation is associated with carotid atherosclerosis in dialysis patients. CREED Investigators. Cardiovascular Risk Extended Evaluation in Dialysis Patients. J Hypertens 2000; 18:1207-1213.

12 Zoccali C, Mallamaci F, Tripepi G: Atherosclerosis in dialysis patients: does Chlamydia pneumoniae infection contribute to cardiovascular damage? Nephrol Dial Transplant 2002; 17(suppl 8):25-28; discussion 40.

13 Stenvinkel P: Interactions between inflammation, oxidative stress, and endothelial dysfunction in end-stage renal disease. J Ren Nutr 2003; 13:144-148.

14 Blacher J, Asmar R, Djane S, London GM, Safar ME: Aortic pulse wave velocity as a marker of cardiovascular risk in hypertensive patients. Hypertension 1999;33:1111-1117. 
15 Davies M, Hruska K: Pathophysiological mechanisms of vascular calcification in endstage renal disease. Kidney Int 2001;60:472479.

-16 Rajasree S, Umashankar PR, Lal AV, Sarma PS, Kartha CC: 1,25-Dihydroxyvitamin D3 receptor is upregulated in aortic smooth muscle cells during hypervitaminosis D. Life Sci 2002; 70:1777-1788.

17 Rebsamen MC, Sun J, Norman AW, Liao JK: 1alpha,25-dihydroxyvitamin D3 induces vascular smooth muscle cell migration via activation of phosphatidylinositol 3-kinase. Circ Res 2002;91:17-24.

18 McCarty MF: Secondary hyperparathyroidism promotes the acute phase response - A rationale for supplemental vitamin D in prevention of vascular events in the elderly. Med Hypotheses 2005; 64:1022-1026.

19 Timms PM, Mannan N, Hitman GA, et al: Circulating MMP9, vitamin D and variation in the TIMP-1 response with VDR genotype: mechanisms for inflammatory damage in chronic disorders? QJM 2002;95:787-796.

-20 Park CW, Oh YS, Shin YS, et al: Intravenous calcitriol regresses myocardial hypertrophy in hemodialysis patients with secondary hyperparathyroidism. Am J Kidney Dis 1999;33: 73-81.

-21 McGonigle RJ, Fowler MB, Timmis AB, et al: Uremic cardiomyopathy: potential role of vitamin D and parathyroid hormone. Nephron 1984;36:94-100.

22 Resnick LM, Muller FB, Laragh JH: Calciumregulating hormones in essential hypertension. Relation to plasma renin activity and sodium metabolism. Ann Intern Med 1986;105:649_ 654.

-23 Burgess ED, Hawkins RG, Watanabe M: Interaction of 1,25-dihydroxyvitamin D and plasma renin activity in high renin essential hypertension. Am J Hypertens 1990;3:903-905.
24 Li Y, Kong J, Wei M, et al: 1,25-Dihydroxyvitamin $D(3)$ is a negative endocrine regulator of the renin-angiotensin system. J Clin Invest 2002; 110:229-238.

25 Pfeifer M, Begerow B, Minne HW, et al: Effects of a short-term vitamin $\mathrm{D}(3)$ and calcium supplementation on blood pressure and parathyroid hormone levels in elderly women. J Clin Endocrinol Metab 2001;86:1633-1637.

26 Xiang W, Kong J, Chen S, et al: Cardiac hypertrophy in vitamin $\mathrm{D}$ receptor knockout mice role of the systemic and cardiac renin-angiotensin systems. Am J Physiol Endocrino Metab 2005;266:E125-E132.

27 Siren AL, Fratelli M, Brines M, et al: Erythropoietin prevents neuronal apoptosis after cerebral ischemia and metabolic stress. Proc Natl Acad Sci USA 2001;98:4044-4049.

-28 Cerami A: Beyond erythropoiesis: novel applications for recombinant human erythropoietin. Semin Hematol 2001;38:33-39.

29 Fineberg N, Weinberg M: The influence of age on renal function and renin and aldosterone response to sodium-volume expansion and contraction in normotensive and mildly hypertensive humans. Am J Hypertens 1992;5:520 528.

30 McCullough PA, Sandberg KR, Yee J, Hudson MP: Mortality benefit of angiotensin-converting enzyme inhibitors after cardiac events in patients with end-stage renal disease. J Renin Angiotensin Aldosterone Syst 2002;3:188191.

31 Quinkler M, Zehnder D, Eardley KS, et al: Increased expression of mineralocorticoid effector mechanisms in kidney biopsies of patients with heavy proteinuria. Circulation 2005;112: 1435-1443.
32 McCarty MF: Acid-base balance may influence risk for insulin resistance syndrome by modulating cortisol output. Med Hypotheses 2005; 64:380-384.

33 Zoccali C, Mallamaci F, Tripepi G: Traditional and emerging cardiovascular risk factors in end-stage renal disease. Kidney Int Suppl 2003:S105-S110.

-34 Zoccali C: Endothelial damage, asymmetric dimethylarginine and cardiovascular risk in endstage renal disease. Blood Purif 2002;20:469_ 472.

35 Tripepi G, Mallamaci F, Zoccali C: Inflammation markers, adhesion molecules, and allcause and cardiovascular mortality in patients with ESRD: searching for the best risk marker by multivariate modeling. J Am Soc Nephrol 2005;16(suppl 1):S83-S88.

36 Shoji T, Shinohara K, Kimoto E, et al: Lower risk for cardiovascular mortality in oral 1alpha-hydroxy vitamin D3 users in a haemodialysis population. Nephrol Dial Transplant 2004; 19:179-184.

37 Teng M, Wolf M, Lowrie E, et al: Survival of patients undergoing hemodialysis with paricalcitol or calcitriol therapy. N Engl J Med 2003; 349:446-456.

38 Levin A: The relationship of haemoglobin level and survival: direct or indirect effects? Nephrol Dial Transplant 2002;17:8-13.

39 Brenner BM, Cooper ME, de Zeeuw D, et al: Effects of losartan on renal and cardiovascular outcomes in patients with type 2 diabetes and nephropathy. N Engl J Med 2001;345:861869.

40 Foley RN, Herzog CA, Collins AJ: Blood pressure and long-term mortality in United States hemodialysis patients: USRDS Waves 3 and 4 Study. Kidney Int 2002;62:1784-1790.

-41 Efrati S, Zaidenstein R, Dishy V, et al: ACE inhibitors and survival of hemodialysis patients. Am J Kidney Dis 2002;40:1023-1029. 